CLINICAL RESEARCH ARTICLE

\title{
Clinical phenotypes of acute kidney injury are associated with unique outcomes in critically ill septic children
}

\author{
Rajit K. Basu (D) ${ }^{1}$, Richard Hackbarth ${ }^{2}$, Scott Gillespie ${ }^{3}$, Ayse Akcan-Arikan ${ }^{4}$, Patrick Brophy ${ }^{5}$, Sean Bagshaw ${ }^{6}$, Rashid Alobaidi ${ }^{7}$ and
} Stuart L. Goldstein ${ }^{8}$

BACKGROUND: Assessment of acute kidney injury (AKI) in septic patients remains imprecise. In adults, the classification of septic patients by clinical AKI phenotypes (severity and timing) demonstrates unique associations with patient outcome vs. broadly defined AKI.

METHODS: In a multinational prospective observational study, AKI diagnosis in critically ill septic children was stratified by duration (transient vs. persistent) and severity (mild vs. severe by creatinine change and urine output). The outcomes of interest were mortality and intensive care unit resource complexity at 28 days.

RESULTS: Seven hundred and fifty-seven septic children were studied (male 52.7\%, age 4.6 years (1.5-11.9)). Mortality (overall $12.1 \%)$ was different between severe AKI and mild AKI (18.3 vs. 4.4\%, $p<0.001)$ as well as intensive care unit (ICU) complexity (overall 34.5\%, 45 vs. 21.7\%, $p<0.001)$. Patients with Persistent AKI had fewer ICU-free days $(17(7,21)$ vs. $24(17,26), p<0.001)$ and higher ICU complexity ( 52.8 vs. $22.9 \%, p=0.002$ ) than transient AKI, even after exclusion of patients with early mortality. AKI phenotypes incorporating temporal and severity data correlate with unique survival (range 4.4-21.6\%) and ICU-free days (range of 15-25 days)

CONCLUSIONS: The outcome of septic children with AKI changes by clinical phenotype. Our findings underscore the importance of prognostic enrichment in sepsis and AKI for the purpose of trial design and patient management.

Pediatric Research (2021) 90:1031-1038; https://doi.org/10.1038/s41390-021-01363-3

\section{IMPACT:}

- Although AKI occurs commonly in patients with sepsis (S-AKI), outcomes for children with S-AKI varies based on the severity and timing of the AKI.

- Existing S-AKI pediatric data utilize a broad singular definition of kidney injury. Increasing the precision of AKI classification results in a new understanding of how S-AKI associates with patient outcome.

- A refined classification of S-AKI identifies subgroups of children, making possible a targeted and a personalized medicine approach to S-AKI study and management.

\section{INTRODUCTION}

Sepsis is the most common cause of acute kidney injury (AKI). ${ }^{1-6}$ In both resource-rich and resource-limited settings, sepsis and AKI individually are associated with poor patient outcome, increasing the utilization of hospital and intensive care unit (ICU) resources (including length of stay (LOS)) and the probability of death. ${ }^{2,4}$ Sepsis with AKI (S-AKI) appears to be associated with worse outcomes over either syndrome alone. Compared to sepsis or AKI in isolation, patients with S-AKI have increased hospital and ICU LOS, increased overall costs, higher rates of mortality, and in survivors, increased rates of long-term disability and reduced quality of life. ${ }^{1,2,4,5,7-12}$ The available data in pediatrics, although less in terms of overall size of patients reported, are consistent with adult data-AKI in the context of sepsis appears to be a negative prognostic condition. The importance of AKI in sepsis is not just tied to mortality, but also to chronic kidney disease and neurologic disability in surviving children..$^{5,9,13-16}$

Adoption of AKI definition criteria has facilitated the delineation of AKI epidemiology in the context of critical illness. Most datasets have reported $\mathrm{AKI}$ incidence and prevalence using broad inclusion criteria with regards to timing and disease severity. For the purpose of describing epidemiology, the majority of data report AKI (alone or concurrent with other illnesses) as any degree of AKI incurred in the first 7 days of ICU course-and this adjudication denoted in a binary fashion as the presence of AKI is analyzed for association with patient outcome. ${ }^{2,3,5,14}$ Recent consensus

\footnotetext{
${ }^{1}$ Division of Pediatric Critical Care, Children's Healthcare of Atlanta, Emory University, Atlanta, GA, USA; ${ }^{2}$ Division of Pediatric Critical Care, Helen DeVos Children's Hospital, Spectrum Health, Grand Rapids, MI, USA; ${ }^{3}$ Department of Pediatrics, Division of Biostatistics, Emory University, Atlanta, GA, USA; ${ }^{4}$ Department of Pediatrics, Sections of Critical Care and Nephrology, Baylor College of Medicine, Texas Children's Hospital, Houston, TX, USA; ${ }^{5}$ Department of Pediatrics, Golisano Children's Hospital, Fort Myers, FL, USA;

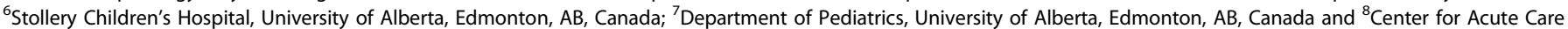
Nephrology, Cincinnati Children's Hospital, Cincinnati, OH, USA

Correspondence: Rajit K. Basu (Rajit.basu@choa.org)
}

Received: 22 July 2020 Revised: 4 November 2020 Accepted: 25 December 2020

Published online: 2 February 2021 
statements recommend, however, adjudicating the impact of AKI with patient outcome after stratification by severity stage and duration of injury. ${ }^{17-20}$ Specifically, this stratification involves staging AKI severity by both creatinine changes from baseline and urine output stage using the Kidney Disease: Improving Global Outcomes (KDIGO) staging criteria and, simultaneously, delineation of the timing of AKI by duration (transient or persistent after $48 \mathrm{~h}$ of initial confirmation of injury). ${ }^{17}$ Application of these definitions in pediatric patients has not occurred. Specifically, there are no reports in septic children describing how staging AKI by severity or duration of injury impacts the associations with patient outcome, although several adult population analyses suggest that such stratification demonstrates unique associations with patient outcome depending on the type of AKI. ${ }^{1,4}$

We previously completed a broad-scale, multinational assessment of AKI in critically ill children (NCT01987921). ${ }^{14,21}$ Interrogation of this population offers us the opportunity to explore the relationship between clinical phenotype of S-AKI and outcome in children. In this report, we describe the stratification of S-AKI by temporal and severity phenotypes, as delineated by consensus guideline statements.

\section{METHODS}

Population

We have previously described the population of patients enrolled and studied in the Assessment of Worldwide AKI, Renal Angina and Epidemiology in Children (AWARE, NCT01987921). Briefly, in one calendar year, 32 unique centers across the world enrolled children and young adults consecutively over a 3-month time span who met the inclusion and exclusion criteria. The most significant inclusion criterion was expected to stay $>48 \mathrm{~h}$. Patients aged 3 months to 25 years were included. The most significant exclusion criterion was pre-existing chronic kidney disease. The primary purpose of AWARE was to delineate the epidemiology and outcomes of AKI in critically ill children, adolescents, and young adults across all admission criteria and illness (NCT01987921). ${ }^{21}$

\section{Sepsis}

Sepsis was denoted on the day of ICU admission (Day 0) using the pediatric Surviving Sepsis Guidelines Criteria and was adjudicated by the site investigator representing each center participating in AWARE.

\section{Sepsis-associated AK}

AKI was staged across the first 7 days of the ICU course. Broadly classified S-AKI, henceforth known as "Any AKI," was identified by the maximum KDIGO stage in the first 7 days using change from baseline creatinine or urine output classification. ${ }^{6,20}$

\section{S-AKI clinical phenotypes}

AKI classifications, or clinical phenotypes, were created by adjudicating for injury severity and injury as outlined by consensus recommendations. The severity of AKI was staged using the worse of serum creatinine change from baseline or urine output on the day of staging. Under stage 1 was denoted "No AKI," stage 1 was termed "Mild AKI," stages 2 and 3 was termed "Severe AKI." The duration of AKI was adjudicated using a temporal phenotype after $48 \mathrm{~h}$. Transient AKI was defined as AKI detected from Days 0 to 2 with subsequent return to AKI stage 0 on Day 3 (i.e., $72 \mathrm{~h}$ ). AKI lasting beyond $48 \mathrm{~h}$ ( $\geq 72 \mathrm{~h}$ ) was termed Persistent-AKI. Patients with de novo AKI after $48 \mathrm{~h}$ were not counted in this analysis. Combining the severity and temporal classifications allowed separation into more granular phenotypes: Mild-transient, Mild-persistent, Severe-transient, and Severepersistent AKI.

\section{Exposures}

Due to the heterogeneity of the centers participating in AWARE and the pragmatic nature of the study, severity of illness (SOI) scores tabulated were the scores used by the individual site itself. Three different SOI scores were used in this study: Pediatric Risk of Mortality-3 (PRISM-3), pediatric logistic organ dysfunction (PELOD) score, and Pediatric Index of Mortality-2 (PIM-2). To control for the inherent variation between the different SOI scores used, patients were tiered into quartiles based on their respective SOI scores; an SOI score $>75$ th percentile and corresponding mortality risk score for any of the three severity measures were used to normalize illness severity and tabulated as a binary metric across the population. The primary condition necessitating ICU admission was delineated into broad classifications as per the initial protocol for the AWARE study. Comorbid conditions outside sepsis and a transplant history were detailed but interpreted as a binary function in this analysis. Examples of the comorbid conditions can be found in the description of the AWARE protocol and detailed in the primary epidemiologic report. ${ }^{14,21}$ Transplant history was a separate exposure variable and included solid organ or bone marrow transplantation.

\section{Outcomes}

The primary outcome of interest was mortality censored at 28 days. Secondary outcomes were also censored at 28 days and included the use of mechanical ventilation, continuous renal replacement (CRRT), extracorporeal membrane oxygenation (ECMO), ICU-free days (28 days minus LOS), and ventilator-free days ( 28 days minus the duration of mechanical ventilation). For each of ICU- and ventilator-free days, mortality was defaulted to 0 . A composite assessment of ICU was also included (denoted as complex ICU course) and defined as a composite of mortality, <14 ICU-free days, <21 ventilator-free days, use of CRRT, or use of ECMO. CRRT was separated from AKI severity to understand the contribution of this support therapy with outcome in the setting of AKI. A patient qualified as having a "complex ICU course" by experiencing any one of these individual metrics. We performed separate analyses excluding patients with early mortality within $48 \mathrm{~h}$ ) to further refine temporal phenotypic associations with outcome.

\section{Statistical analysis}

All analyses were performed in SAS v.9.4 (Cary, NC) and R v.3.6 (Vienna, Austria), and statistical significance was evaluated at the 0.05 threshold, unless otherwise noted. Patient demographics and clinical and outcome characteristics were summarized by relevant sepsis and AKI categories using medians and interquartile ranges for continuous variables and frequencies and percentages for discrete characteristics. Omnibus differences in continuous data between study groups were tested using Kruskal-Wallis tests; concurrently, categorical data were tested for overall differences using $x^{2}$ tests of independence or Fisher's exact tests, as appropriate. When omnibus tests were significant, pairwise tests were carried out on study groups of interest, and the significance level was adjusted to the corresponding Bonferroni threshold (e.g., $0.05 / 3$ pairwise comparisons $=0.017$ ). Pairwise differences in outcome characteristics, such as ventilation use, CRRT, and ECMO, were further assessed after adjustment for SOI scores, with adjusted $p$ values calculated using generalized linear regression procedures: linear regression for continuous outcomes and logistic regression for discrete. The primary outcome for this study was $28-$ day mortality. Complex ICU course was also computed by the individual components listed earlier. Treating each outcome dichotomously, binary logistic regression was employed for each case to determine significant bivariable associations. Informed by these bivariable results, stepwise regression procedures were employed to build the final models. In each multivariable 
regression case, the primary exposure was S-AKI status. Finally, time-to-event procedures, in the form of competing risks analyses (CRAs), were performed to model the probability over time of each of two mutually exclusive endpoints: (1) 28-day mortality and (2) 28-day ICU discharge; the remaining patients being alive without ICU discharge. Results from these analyses are reported via cumulative incidence curves and $95 \%$ confidence intervals and reported at 28 days follow-up. S-AKI status differences between cumulative incidence curves for each endpoint were tested using Gray's tests.

\section{RESULTS}

Population

A total of 4898 patients were identified from the original data for sepsis and AKI classifications (Supplementary Table 1). A total of 757 (15.5\% of overall patient cohort) patients classified with sepsis at the time of enrollment are discussed in this analysis. In the septic population, $40.6 \%$ (307) were classified as Any AKI in the first 7 days of ICU course. Compared to No AKI, patients with Any AKI had lower weight at ICU admission $(p=0.016)$, but there were no other significant demographic differences. On ICU admission, however, patients with Any AKI were sicker than No-AKI patients, carrying a higher incidence of shock as an ICU admitting diagnosis and a more frequent history of transplant (both $p<0.001$ ). Any-AKI patients had a higher mortality rate $(12.1$ vs. $4.7 \%, p<0.001)$ and increased utilization of ICU technology such as mechanical ventilation $(p=0.017)$ and CRRT $(p<0.001)$. After adjustment for significant bivariable associations, the classification of Any AKI remained independently associated with higher odds of mortality relative to No AKI (Table 1). In addition, overall ICU complexity was increased for patients with Any AKI compared to No AKI in the setting of sepsis (Supplementary Table 2).

Severity of AKI is associated with outcome in Severe AKI Of the 307 patients with Any AKI, 45\% (138) were classified as Mild AKI, while 55\% (169) were denoted as Severe AKI (Table 2). SevereAKI patients were sicker than Mild- and No-AKI patients (who demonstrated no difference in SOI scores), but other demographic characteristics between the groups were similar. After adjustment for SOI, Severe AKI (but not Mild AKI) was significantly associated with 28 -day mortality compared to No AKI (18.3 vs. $4.7 \%, p=$ 0.001) (Supplementary Table 3). Severe-AKI patients also had a higher incidence of complex ICU course $(p<0.001)$, with significantly fewer mechanical ventilation free days $(p<0.001)$, fewer ICU-free days $(p<0.001)$, and a higher utilization of CRRT ( $p$ $<0.001$ ). After correction for illness severity, mortality rate was not significantly different between Severe- and Mild-AKI patients, but the incidence of CRRT utilization ( 16.6 vs. $0.7 \%, p=0.006)$, 28-day ICU-free days $\left(19^{7,22}\right.$ vs. $\left.23,{ }^{17,23} p=0.007\right)$, and complex ICU outcome (45 vs. $21.7 \%, p<0.001$ ) remained significantly different.

Duration of AKI is associated with outcome in Severe AKI Duration of injury data was available on 724 (95.6\%) of the septic patients. In these patients, $37.8 \%(274)$ had Any AKI. Transient AKI was present in $60.6 \%$ (166), while $39.4 \%$ (108) had Persistent AKI (Table 3). No-AKI patients were demographically similar to the Transient-AKI patients and demonstrated similar SOI. In contrast, the Persistent-AKI patients were significantly sicker than No AKI (but not Transient AKI). After adjustment for covariates including illness severity, Persistent-AKI patients demonstrated a higher incidence of complex ICU outcome ( 52.8 vs. $16.9 \%, p<0.001)$ with fewer mechanical ventilation-free days $(p<0.001)$, fewer ICU-free days $(p<0.001)$, and higher CRRT utilization $(p<0.001)$ vs. No-AKI patients (Supplementary Table 4). In addition, the patients with Persistent AKI had a higher incidence of complex ICU course than the Transient-AKI patients (52.8 vs. $22.9 \%, p=0.001)$ and fewer ICU-free days $(p<0.001)$. After exclusion of the 17 patients with death within $48 \mathrm{~h}$, the comparison of outcome and ICU resource utilization between Transient AKI and Persistent AKI demonstrated significant differences in CRRT utilization $(p=0.005)$, and 28-day mechanical ventilation and ICU duration $(p=0.002$ and $<0.001$, respectively) (Supplementary Tables 5 and 6 and Supplementary Figs 1 and 2).

\section{S-AKI sub-phenotypes demonstrate unique associations with outcome}

A combination of AKI stage and duration classifications facilitated a further sub-phenotypic analysis; 274 patients with data were available. Of the 274 septic patients with Any AKI, 33.6\% had Mildtransient, $11.7 \%{ }^{24}$ had Mild-persistent, $27 \%$ had Severe-transient AKI, and $27.7 \%$ had Severe-persistent AKI (Supplementary Table 7). The rate of death varied across the clinical AKI sub-phenotypes (4.4-21.6\%) (Fig. 1). Complex ICU course demonstrated a similarly disparate range of incidences across the sub-phenotypes (15.2-54\%), compared to No AKI (16.9\%). The patients with Severe-persistent AKI were sicker than the Mild-transient AKI and demonstrated worsened outcome and unadjusted and adjusted for SOI score. Despite no difference in illness severity or mortality rate compared to Mild-transient, patients with Mild-persistent had a higher incidence of mechanical ventilation $(p=0.005)$, fewer ICU-free days $(p<0.001)$, and more complex ICU courses $(p<0.001)$. Similarly, patients with Severe-persistent AKI were demographically similar to Severe-transient AKI patients and had no difference in SOI or mortality but had fewer ICU-free days $\left(18^{7,25}\right.$ vs. $\left.24,{ }^{7,23} p=0.005\right)$ and more complex ICU courses (although not significantly different after SOI adjustment).

Compared to the classification of Any AKI, both mortality rate and ICU-free days varied between AKI sub-phenotypes. Mortality varied significantly between AKI sub-phenotype (Fig. 1 and Supplementary Table 8). While the cumulative probability of ICU discharge at 28 days was $85.1 \%$ for Any-AKI patients, the cumulative probabilities of 28-day ICU discharge ranged from 76.1 to $93.2 \%$ based on sub-phenotype (Fig. 2). Significant differences existed between Mild-transient AKI and all other AKI sub-phenotypes (Supplementary Tables 9 and 10). A correction to study AKI managed with CRRT vs. Severe AKI demonstrated significant bivariate associations between SOI (shock diagnosis primarily) and ICU complexity, including mortality, 28-day ICU duration, and mechanical ventilation use $(p<0.001$ for all) (Supplementary Table 11). Finally, ICU resource utilization based on mortality and discharge were analyzed excluding early mortality (see Transient vs. Persistent-AKI results) and demonstrated significantly worse outcomes for the Mild-persistent and Severe-persistent AKI phenotypes (Supplementary Table 12), including for Severe-persistent compared to Severe-transient AKI (Supplementary Table 13).

\section{DISCUSSION}

Sepsis and AKI affect a significant proportion of critically ill patients. Recent epidemiologic data indicate $33-50 \%$ of critically ill patients suffer from sepsis and $\sim 25 \%$ suffer from AKI. ${ }^{2,6,14}$ Across epidemiologic datasets-from neonates to adults, these patients have worse outcomes than those without sepsis or AKI, respectively. The prevailing data suggest patients with concurrent sepsis and AKI (S-AKI) suffer a higher utilization of ICU technology, prolonged hospital and ICU course, and increased rates of mortality. The data from our multinational cohort of critically ill children and young adults reinforce that patients with S-AKI suffer worse outcomes. However, our analysis is unique from the existing data. Our data demonstrate that not all S-AKI is equivalent. This is the first report in a large population of pediatric population, to our knowledge, to incorporate contemporary recommendations for clinical adjudication of AKI diagnosis. Although our findings validate the findings in adult septic patients, ${ }^{1,2,4,10,25}$ we 
Table 1. 28-Day mortality in septic patients $(N=757)$.

\begin{tabular}{|c|c|c|c|c|c|c|}
\hline \multirow[t]{2}{*}{ Category, N (row \%) } & \multirow{2}{*}{$\begin{array}{l}\text { Alive } \\
N=699(92.3 \%)\end{array}$} & \multirow{2}{*}{$\begin{array}{l}\text { Dead } \\
N=58(7.7 \%)\end{array}$} & \multicolumn{2}{|l|}{ Bivariable } & \multicolumn{2}{|l|}{ Multivariable $^{a}$} \\
\hline & & & OR $(95 \% \mathrm{Cl})$ & $P$ value & OR $(95 \% \mathrm{Cl})$ & $P$ value \\
\hline Age at ICU admission (years) ${ }^{\mathrm{b}}$ & $4.5(1.4,12.3)$ & $6.1(1.7,13.7)$ & $1.13(0.92,1.40)$ & 0.239 & & \\
\hline \multicolumn{7}{|l|}{ Gender } \\
\hline Male & $365(91.5 \%)$ & $34(8.5 \%)$ & $1.30(0.75,2.23)$ & 0.349 & & \\
\hline Female & $334(93.3 \%)$ & $24(6.7 \%)$ & Reference & & & \\
\hline Weight at ICU admission $(\mathrm{kg})^{\mathrm{b}}$ & $16.1(9.9,38.3)$ & $17(9.1,35)$ & $0.99(0.93,1.04)$ & 0.637 & & \\
\hline Any comorbid conditions & $587(91.4 \%)$ & $55(8.6 \%)$ & $3.50(1.08,11.4)$ & 0.038 & & \\
\hline \multicolumn{7}{|l|}{ Admission diagnosis } \\
\hline Shock & $402(89.7 \%)$ & $46(10.3 \%)$ & $2.83(1.47,5.44)$ & 0.002 & & \\
\hline Medical cardiac & 17 (73.9\%) & $6(26.1 \%)$ & $4.63(1.75,12.2)$ & 0.002 & $6.62(1.43,30.5)$ & 0.016 \\
\hline Respiratory failure & $319(90.4 \%)$ & $34(9.6 \%)$ & $1.69(0.98,2.91)$ & 0.059 & & \\
\hline Post-surgical/minor trauma & $54(94.7 \%)$ & $3(5.3 \%)$ & $0.65(0.20,2.15)$ & 0.482 & & \\
\hline CNS dysfunction & $140(89.2 \%)$ & $17(10.8 \%)$ & $1.66(0.91,3.00)$ & 0.097 & & \\
\hline Pain/sedation management & $13(86.7 \%)$ & $2(13.3 \%)$ & $1.89(0.42,8.56)$ & 0.412 & & \\
\hline PRISM-III & $5(0,11)$ & 149,22 & $1.13(1.07,1.18)$ & $<0.001$ & & \\
\hline PIM-2 & 11,4 & 74,40 & $1.06(1.03,1.08)$ & $<0.001$ & & \\
\hline PELOD & 112,12 & $20.511,32$ & $1.10(1.05,1.16)$ & $<0.001$ & & \\
\hline \multicolumn{7}{|c|}{ Any severity score $>75$ th percentile } \\
\hline Yes & $85(76.6 \%)$ & $26(23.4 \%)$ & $8.16(3.68,18.1)$ & $<0.001$ & $6.12(2.58,14.5)$ & $<0.001$ \\
\hline No & $240(96.4 \%)$ & $9(3.6 \%)$ & Reference & & Reference & \\
\hline History of transplant & $49(87.5 \%)$ & $7(12.5 \%)$ & $1.82(0.79,4.23)$ & 0.163 & & \\
\hline \multicolumn{7}{|l|}{ AKI status } \\
\hline Any AKI & $270(87.9 \%)$ & 37 (12.1\%) & $2.80(1.60,4.89)$ & $<0.001$ & $2.67(1.23,5.77)$ & 0.013 \\
\hline No AKI & $429(95.3 \%)$ & $21(4.7 \%)$ & Reference & & Reference & \\
\hline Mechanical ventilation use & 299 (87.9\%) & $41(12.1 \%)$ & $2.94(1.64,5.29)$ & $<0.001$ & $2.72(1.02,7.23)$ & 0.045 \\
\hline 28-Day MV duration ${ }^{\mathrm{b}}$ & $4.52,8$ & 52,12 & $1.09(0.88,1.36)$ & 0.437 & & \\
\hline CRRT use & $15(48.4 \%)$ & $16(51.6 \%)$ & $17.4(8.04,37.5)$ & $<0.001$ & & \\
\hline ECMO use & $9(75 \%)$ & $3(25 \%)$ & $4.18(1.10,15.9)$ & 0.036 & & \\
\hline 28-Day ICU LOS & $4(3,8)$ & $4.5(2,12)$ & $1.14(0.94,1.38)$ & 0.186 & & \\
\hline
\end{tabular}

ICU intensive care unit, AKI acute kidney injury, PRISM-III Pediatric Risk of Mortality-3, PIM-2 Pediatric Index of Mortality-2, PELOD pediatric logistic organ dysfunction, CNS central nervous system, MV mechanical ventilation, CRRT continuous renal replacement therapy, ECMO extracorporeal membrane oxygenation.

${ }^{\mathrm{a}}$ Multivariable $\mathrm{ROC}=0.81$ and sample size $=360$, due to list-wise deletion.

${ }^{b} \mathrm{OR}$ increments in units of 5 .

demonstrate unique downstream sequelae based on AKI duration and severity. Significant differences in the associations with mortality and ICU resource utilization exist between mild forms of S-AKI and severe forms of AKI. Similarly, transient forms of S-AKI and persistent forms of S-AKI are clinically unique in the long-term disposition of patients.

Improving the precision of AKI diagnosis has been an area of focus. $^{22,23,26-28}$ Although stratification systems to stage AKI by creatinine and urine output have been in place for nearly two decades (and continue to undergo refinement), very little evidence of published data demonstrates the incorporation of stage-based AKI severity into the adjudication of AKI with other injury complexes. That is, while AKI-focused epidemiology has reported extensively on the association between AKI severity stages and outcome, descriptions of AKI in the context of common ICU illnesses such as sepsis, traumatic brain injury, or acute respiratory distress syndrome have not, to our knowledge, incorporated AKI severity staging.,29-32 Our data show that patients with mild AKI in the context of sepsis actually do not have a worsened outcome compared to septic patients without AKI. However, when separated by a duration of AKI, the patients with mild AKI that persists have fewer ICU-free days (adjusted, $p<0.001)$. Septic patients with severe persistent forms AKI have uniquely worse outcomes than septic patients with Mildpersistent AKI. Unlike persistent S-AKI phenotypes, transient forms of AKI, even when severe, are associated with better patient outcome. The granularity of outcomes associated with the subphenotypes should be considered adjacent to the umbrella diagnosis of AKI (Any AKI). Without adjudication for the severity or temporal classifications of AKI, our data indicate roughly one in ten with S-Any AKI (12.9\%) die and one in seven (14.9\%) remain in the ICU at 28 days. After adjudication for S-AKI sub-phenotypes, these proportions change. In the severe sub-phenotypes of S-AKI, between one in five to one in four $(17.1-22.5 \%)$ are dead at 28 days and a slightly higher percentage (20-23.9\%) remain in the ICU. Conversely, in the mild sub-phenotypes of S-AKI, only 1 in 25 may die (4.6\% for Mild-transient S-AKI) and only 1 in 15 (6.8\%) may remain in the ICU at 28 days. After excluding patients who die within $72 \mathrm{~h}$, transient AKI demonstrates a mortality of $5.2 \%$ or 1 in 20. Further, the use of CRRT to manage the multiple sequelae of AKI complicates the understanding of AKI impact. In our analysis of Severe AKI, separation of patients receiving CRRT indicates a significant proportion of AKI-associated outcomes and ICU resource utilization may be driven by the integration of CRRT as 
Clinical phenotypes of acute kidney injury are associated with unique... RK Basu et al.

Table 2. Study characteristics by sepsis and maximum AKI severity $(N=757)$.

\begin{tabular}{|c|c|c|c|}
\hline Category, N (\%) & $\begin{array}{l}\text { No AKI (1) } \\
N=450(59.5 \%)\end{array}$ & $\begin{array}{l}\text { Mild AKI (2) } \\
N=138(18.2 \%)\end{array}$ & $\begin{array}{l}\text { Severe AKI (3) } \\
N=169(22.3 \%\end{array}$ \\
\hline \multicolumn{4}{|l|}{ Demographics/clinical } \\
\hline Age at ICU admission (years) & $3.8(1.4,11.9)$ & $5.6(1.7,12.9)$ & $6.7(1.3,13.1)$ \\
\hline Male gender & $235(52.2 \%)$ & $69(50 \%)$ & $95(56.2 \%)$ \\
\hline Weight at ICU admission $(\mathrm{kg})$ & $15.1(9.7,34)$ & $20(10.5,43.4)$ & $20(9.8,48.6)$ \\
\hline Any comorbid conditions & $377(83.8 \%)$ & $120(87 \%)$ & $145(85.8 \%)$ \\
\hline \multicolumn{4}{|l|}{ Admission diagnosis } \\
\hline Shock & $239(53.1 \%)$ & $85(61.6 \%)$ & $124(73.4 \%)$ \\
\hline Medical cardiac & $13(2.9 \%)$ & $3(2.2 \%)$ & $7(4.1 \%)$ \\
\hline Respiratory failure & $218(48.4 \%)$ & $58(42 \%)$ & $77(45.6 \%)$ \\
\hline Post-surgical/minor trauma & $34(7.6 \%)$ & $7(5.1 \%)$ & $16(9.5 \%)$ \\
\hline CNS dysfunction & $99(22 \%)$ & $28(20.3 \%)$ & $30(17.8 \%)$ \\
\hline $\begin{array}{l}\text { Pain/sedation } \\
\text { management }\end{array}$ & $8(1.8 \%)$ & $4(2.9 \%)$ & $3(1.8 \%)$ \\
\hline History of transplant & $17(3.8 \%)$ & $17(12.3 \%)$ & $22(13 \%)$ \\
\hline PRISM-III & $4(0,10.5)$ & $5(0,10)$ & $10(7,17.5)$ \\
\hline PIM-2 & $1.5(1,4)$ & $1(1,3)$ & $3(1,18)$ \\
\hline PELOD & $10(1,12)$ & 1110,12 & $12(11,21)$ \\
\hline $\begin{array}{l}\text { Any severity score }>75 \text { th } \\
\text { percentile }\end{array}$ & $65(28.4 \%)$ & $13(22 \%)$ & $33(45.8 \%)$ \\
\hline \multicolumn{4}{|l|}{ Outcomes } \\
\hline Mechanical ventilation use & $182(43.3 \%)$ & $59(43.4 \%)$ & $99(59.6 \%)^{*}$ \\
\hline CRRT use & $2(0.4 \%)$ & $1(0.7 \%)$ & $28(16.6 \%)^{* \wedge \wedge}$ \\
\hline ECMO use & $6(1.3 \%)$ & $1(0.7 \%)$ & $5(3 \%)$ \\
\hline 28-Day mortality & $21(4.7 \%)$ & $6(4.4 \%)$ & $31(18.3 \%)^{*}$ \\
\hline 28-Day MV-free days & $24(19,26)$ & $23(19,25)$ & $19(0,24)^{*}$ \\
\hline 28-Day ICU-free days & $24(20,25)$ & $23(17,25)$ & $19(7,24)^{* * \wedge}$ \\
\hline Complex ICU course & $76(16.9 \%)$ & $30(21.7 \%)$ & $76(45 \%)^{*} \wedge$ \\
\hline
\end{tabular}

ICU intensive care unit, AKI acute kidney injury, PRISM-III Pediatric Risk of Mortality-3, PIM-2 Pediatric Index of Mortality-2, PELOD pediatric logistic organ dysfunction, CNS central nervous system, MV mechanical ventilation, CRRT continuous renal replacement therapy, ECMO extracorporeal membrane oxygenation, severity score based on sepsis sample $(N=757)$.

After adjustment for severity of illness (Supplementary Table 3 ), $p$ value $<0.017$ was considered at the Bonferroni threshold $(0.05 / 3)$ for: *No vs. Severe and $\wedge$ Mild vs. Severe. In the appendix, column number in this table is used for reference columns of statistics. The $>75$ th percentile for severity of illness scores data shown are the number of patients with an available SOl score $>75$ th percentile for that entire subgrouping (e.g., No AKI$28.4 \%=65$ of 229 with an SOI score in that group were $>75$ th percentile). Available SOI scores in each group for this table: No AKI: 229 (50.9\%), Mild AKI: 59 (42.7\%), and Severe AKI: 72 (42.6\%).

${ }^{a}$ Of 757 sepsis patients, $N=33$ were excluded due to No AKI within first $48 \mathrm{~h}$ of observation.

part of management. This may suggest distinct phenotypes between Severe-AKI cohorts (those that require CRRT and those who do not), an opportunity for a further and broader investigation. Our data suggest that for a more robust understanding of the downstream associations of AKI in the context of other illnesses, AKI in the context of sepsis should not be simply classified as present or absent at any time in the first 7 days of the ICU course.

Therapeutic options for AKI are altogether missing. Best practice recommendations for AKI management continue to be focused solely on supportive care and mitigation of inciting agents and potential ongoing AKI risk. ${ }^{24,33-35}$ Previous trials testing potential AKI therapy have failed to demonstrate consistent efficacy at resolving $\mathrm{AKI}$ or reducing the severity of injury. Although these trials suffered from a number of limitations, a potentially major source of confounding and barrier to answering whether these interventions were effective are the diagnosis of AKI itself. Most trials included AKI as a catchment diagnosis so that, for instance, a trial in our cohort of nearly 750 septic patients would include Mildtransient AKI together with Severe-persistent AKI. This is
Table 3. Study characteristics by sepsis and AKI duration $(N=724)$.

\begin{tabular}{llll}
\hline Category, $N(\%)^{\text {a }}$ & No AKI (1) & Transient & Persistent \\
& $N=450$ & AKI (2) & AKI (3) \\
& $(62.2 \%)$ & $N=166$ & $N=108$ \\
& & $(22.9 \%)$ & $(14.9 \%)$
\end{tabular}

Demographics/clinical

Age at ICU admission (years)

Male gender

Weight at ICU

admission $(\mathrm{kg})$

Any comorbid

conditions

$3.8(1.4,11.9) 5.5(1.4,12.4) 6.8(1.2,14.3)$

Admission diagnosis

Shock

Medical cardiac

Respiratory failure

$235(52.2 \%) \quad 92(55.4 \%) \quad 59(54.6 \%)$

Post-surgical/

minor trauma

CNS dysfunction

$15.1(9.7,34) \quad 19.4(9.9,45) \quad 20(9.9,48.6)$

$377(83.8 \%) \quad 145(87.4 \%) \quad 92(85.2 \%)$

Pain/sedation

management

History of transplant

PRISM-III

PIM-2

PELOD

Any severity score

$>75$ th percentile

Outcomes

Mechanical

ventilation use

CRRT use

ECMO use

28-Day mortality

28-Day MV-free days

28-Day ICU-free days

$\begin{array}{ccc}13(2.9 \%) & 5(3 \%) & 4(3.7 \%) \\ 218(48.4 \%) & 61(36.8 \%) & 55(50.9 \%) \\ 34(7.6 \%) & 15(9 \%) & 7(6.5 \%) \\ & & \\ 99(22 \%) & 36(21.7 \%) & 16(14.8 \%) \\ 8(1.8 \%) & 6(3.6 \%) & 0(0 \%)\end{array}$

$17(3.8 \%) \quad 19(11.5 \%) \quad 16(14.8 \%)$

$4(0,10.5) \quad 7.5(2,13) \quad 10(7,14)$

$1.5(1,4) \quad 1(1,5) \quad 3(1,6)$

$10(1,12) \quad 11(10,12) \quad 12(11,22)$

$65(28.4 \%) \quad 20(32.8 \%) \quad 21(38.9 \%)$

Complex ICU course

$182(43.3 \%) \quad 68(42.2 \%) \quad 72(66.7 \%)^{*}$

$2(0.4 \%) \quad 7(4.2 \%) \quad 21(19.4 \%)^{*}, \wedge$

$6(1.3 \%) \quad 2(1.2 \%) \quad 4(3.7 \%)$

$21(4.7 \%) \quad 20(12.1 \%) \quad 15(13.9 \%)$

$24(19,26) \quad 23(11,26) \quad 19(3,23)^{*}$

$24(20,25) \quad 24(17,26) \quad 17(7,21)^{*, \wedge}$

$76(16.9 \%) \quad 38(22.9 \%) \quad 57(52.8 \%)^{*}, \wedge$

ICU intensive care unit, AKI acute kidney injury, PRISM-III Pediatric Risk of Mortality-3, PIM-2 Pediatric Index of Mortality-2, PELOD pediatric logistic organ dysfunction, CNS central nervous system, $M V$ mechanical ventilation, $C R R T$ continuous renal replacement therapy, ECMO extracorporeal membrane oxygenation, severity score based on sepsis sample $(N=757)$.

After adjustment for univariates (Supplementary Table 4), $p$ value $<0.017$ considered at the Bonferroni threshold $(0.05 / 3)$ for: ${ }^{*}$ No vs. Persistent and $\wedge$ Transient vs. Persistent. In the appendix, column number in this table is used for reference columns of statistics. The $>75$ th percentile for the severity of illness scores data shown are the number of patients with an available SOI score $>75$ th percentile for that entire subgrouping (e.g., No $\mathrm{AKI}-28.4 \%=65$ of 229 with an SOI score in that group were $>75$ th percentile. Available SOI scores in each group for this table: No AKI: 229 (50.9\%), Transient AKI: 61 (36.7\%), and Severe AKI: 54 (50\%).

${ }^{a}$ Of 757 sepsis patients, $N=33$ were excluded due to No AKI within first $48 \mathrm{~h}$ of observation.

problematic as our data show that these patients are different. A previous post hoc analysis of these data validated the renal angina index scoring system to identify the varying degrees of risk for the prediction of Severe-persistent AKI and for targeting biomarker testing. ${ }^{26,29,30,36-40}$ Predictive and prognostic enrichment in S-AKI that we are describing has been mirrored in recent biomarker-based predictive models in children. ${ }^{29,41,42}$ Diagnostic refinement of $\mathrm{AKI}$ using risk stratification, biomarkers, and clinical 

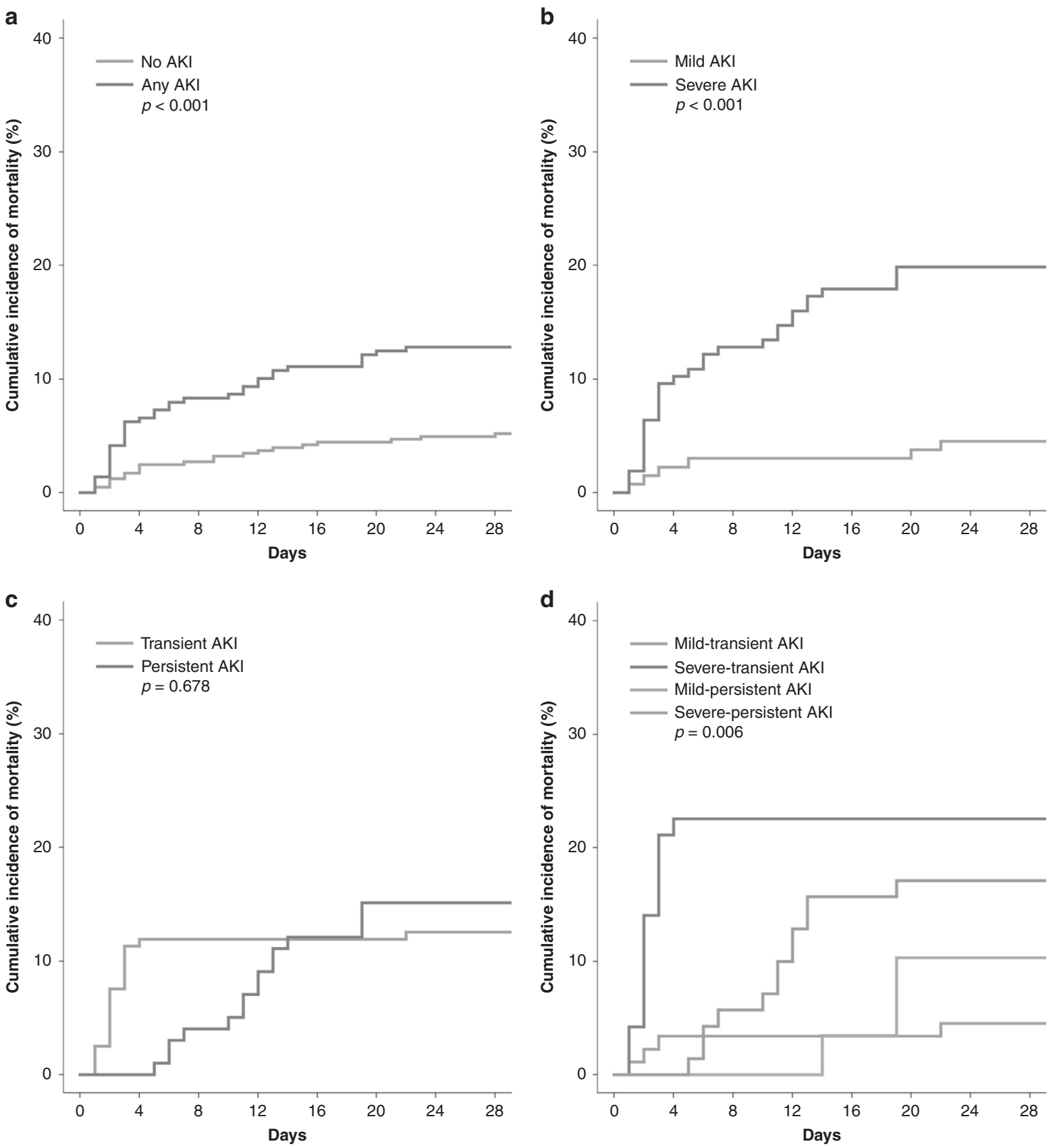

Fig. 1 28-Day mortality cumulative incidence based on sepsis-AKI sub-phenotype. Cumulative incidence plots of mortality as calculated using a broad-based diagnosis No AKI vs. Any AKI, b AKI stratified by severity, c AKI stratified by duration, and d AKI was sub-phenotyped by the combination of severity and duration.

classifications may facilitate the separation of S-AKI populations for targeted therapeutic trial design. In pediatric populations, characterization of the unique aspects of illness in children incorporated into management and trial design facilitates the ability to compare populations of equal risk (for the determination of management efficacy of a given treatment option, for instance). Given the poor patient outcomes in S-AKI, we believe this approach should be explored further.

Our study is notable for its unique strengths and limitations. As stated previously, we believe this is the first large data set to explore the ramifications of a refined diagnostic approach to AKI in the setting of sepsis. The data come from one of the largest and most geographically diverse populations of critically ill children and young adults in the published literature. The analysis, however, is a post hoc analysis and therefore subjected to the limitations inherent with a retrospective analysis. Our associative findings cannot be construed as causal as the AWARE study was designed as a prospective observational study for AKI epidemiology and not interventional, particularly for sepsis. The definition of sepsis used was based on existing pediatric sepsis criteria and we acknowledge that the recently updated criteria have been released. We did not include any biological markers or laboratory data in our definition of AKI subtype classification, although a separate analysis of our data is currently in process for this purpose. In our analyses between sub-phenotypes, to facilitate a correction for illness severity, we created a metric of $>75 \%$ percentile of severity score to normalize the center-specific illness severity scores used. Missing SOI scores may have affected the 

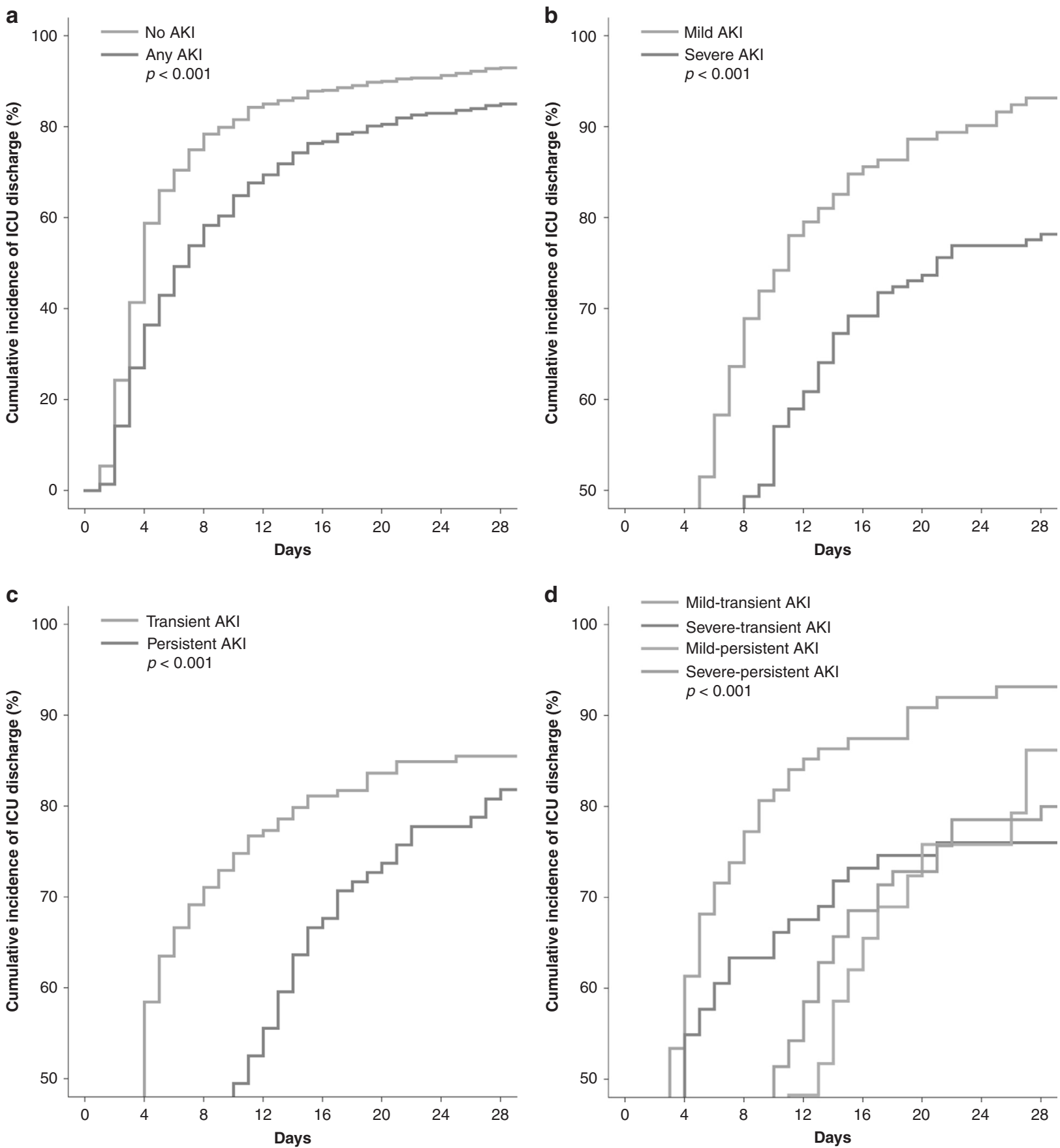

Fig. 2 28-Day ICU discharge cumulative incidence based on sepsis-AKI sub-phenotype. Cumulative incidence plots of rates of discharge from the ICU at 28 days as calculated using a broad-based diagnosis of No AKI vs. Any AKI, b AKI stratified by severity, c AKI stratified by duration, and $\mathbf{d}$ AKI was sub-phenotyped by the combination of severity and duration.

significance of associations after adjustment as many of the unadjusted differences were statistically significant with large effect sizes or absolute differences in percentages, duration of time (e.g., LOS, MV duration), but lost significance after adjustment. However, the initial trial was conducted on a pragmatic basis and the individual centers were allowed the freedom to enter (or not) the SOl score of choice for their given institution. This flexibility facilitated the capture of what was at the time the largest to date prospective data set of critically ill children. Finally, the composite outcome of "complex ICU outcome" has not been previously been validated, although the individual parameters included are all included in the standard reports of ICU outcomes.

\section{CONCLUSION}

In summary, the outcome of septic patients with kidney injury varies by the phenotype of AKI. Sub-phenotypic classification of AKI by severity and temporal characteristics is imminently feasible and novel stratification and diagnostic techniques may be able to identify these subtypes early in the ICU course. The identification of unique clinical AKI targets is, therefore, possible. Our data 
suggest further exploration of the sub-phenotypic approach to AKI is warranted.

\section{ACKNOWLEDGEMENTS}

We acknowledge the efforts of the AWARE contributors and the coordination of the data conducted by Cincinnati Children's Hospital Center for Acute Care Nephrology.

\section{AUTHOR CONTRIBUTIONS}

R.K.B: concept and design, data acquisition, analysis, interpretation, drafting, and final approval. S.G.: concept and design, analysis, interpretation, drafting, and final approval. R.H.: concept and design, analysis, interpretation, drafting, and final approval. A.A.-A.: concept and design, drafting, and final approval. P.B.: interpretation, drafting, and final approval. S.B.: interpretation, drafting, and final approval. R.A.: interpretation, drafting, and final approval. S.L.G.: concept and design, data acquisition, analysis, interpretation, drafting, and final approval.

\section{ADDITIONAL INFORMATION}

Supplementary information The online version contains supplementary material available at https://doi.org/10.1038/s41390-021-01363-3.

Competing interests: The authors declare no competing interests.

Publisher's note Springer Nature remains neutral with regard to jurisdictional claims in published maps and institutional affiliations.

\section{REFERENCES}

1. Poston, J. T. \& Koyner, J. L. Sepsis associated acute kidney injury. BMJ 364, k4891 (2019).

2. Hoste, E. A. J. et al. Global epidemiology and outcomes of acute kidney injury. Nat. Rev. Nephrol. 14, 607-625 (2018).

3. Santos, R. P. D., Carvalho, A. R. S., Peres, L. A. B., Ronco, C. \& Macedo, E. An epidemiologic overview of acute kidney injury in intensive care units. Rev. Assoc. Med. Bras. 65, 2019 (1992).

4. Peerapornratana, S., Manrique-Caballero, C. L., Gómez, H. \& Kellum, J. A. Acute kidney injury from sepsis: current concepts, epidemiology, pathophysiology, prevention and treatment. Kidney Int. 96, 1083-1099 (2019).

5. Alobaidi, R., Basu, R. K., Goldstein, S. L. \& Bagshaw, S. M. Sepsis-associated acute kidney injury. Semin. Nephrol. 35, 2-11 (2015).

6. Susantitaphong, P. et al. World incidence of AKI: a meta-analysis. Clin. J. Am. Soc. Nephrol. 8, 1482-1493 (2013).

7. Schuler, A. et al. The impact of acute organ dysfunction on long-term survival in sepsis. Crit. Care Med. 46, 843-849 (2018).

8. Schortgen, F. \& Asfar, P. Update in sepsis and acute kidney injury 2014. Am. J. Respir. Crit. Care Med. 191, 1226-1231 (2015).

9. Stanski, N. L., Cvijanovich, N. Z., Fitzgeral, J. C., Bigham, M. T. \& Wong, H. R., Investigators GoPSS. Severe acute kidney injury is independently associated with mortality in children with septic shock. Intens. Care Med. 46, 1050-1051 (2020).

10. Peerapornratana, S. et al. Sepsis-associated acute kidney disease. Kidney Int. Rep. 5, 839-850 (2020).

11. Wiersema, R. et al. Two subphenotypes of septic acute kidney injury are associated with different 90-day mortality and renal recovery. Crit. Care 24, 150 (2020).

12. Zarbock, A., Gomez, H. \& Kellum, J. A. Sepsis-induced acute kidney injury revisited: pathophysiology, prevention and future therapies. Curr. Opin. Crit. Care 20, 588-595 (2014).

13. Basu, R. K. Acute kidney injury in hospitalized pediatric patients. Pediatr. Ann. 47, e286-e291 (2018).

14. Kaddourah, A., Basu, R. K., Bagshaw, S. M., Goldstein, S. L. \& Investigators, A. Epidemiology of acute kidney injury in critically ill children and young adults. $N$. Engl. J. Med. 376, 11-20 (2017).

15. Fitzgerald, J. C. et al. Acute kidney injury in pediatric severe sepsis: an independent risk factor for death and new disability. Crit. Care Med. 44, 2241-2250 (2016).
16. Hessey, E., Perreault, S., Dorais, M., Roy, L. \& Zappitelli, M. Acute kidney injury in critically ill children and subsequent chronic kidney disease. Can. J. Kidney Health Dis. 6, 2054358119880188 (2019).

17. Chawla, L. S. et al. Acute kidney disease and renal recovery: consensus report of the Acute Disease Quality Initiative (ADQI) 16 Workgroup. Nat. Rev. Nephrol. 13, 241-257 (2017).

18. Matejovic, M. et al. Renal hemodynamics in AKI: in search of new treatment targets. J. Am. Soc. Nephrol. 27, 49-58 (2016).

19. Thomas, M. E. et al. The definition of acute kidney injury and its use in practice. Kidney Int. 87, 62-73 (2015).

20. Ostermann, M. Diagnosis of acute kidney injury: Kidney Disease Improving Global Outcomes criteria and beyond. Curr. Opin. Crit. Care 20, 581-587 (2014).

21. Basu, R. K. et al. Assessment of worldwide acute kidney injury, renal angina and epidemiology in critically ill children (AWARE, A Prospective Study to Improve Diagnostic Precision. J. Clin. Trials 5, 222 (2015).

22. McMahon, B. A. et al. Biomarker predictors of adverse acute kidney injury outcomes in critically ill patients: The Dublin Acute Biomarker Group Evaluation Study. Am. J. Nephrol. 50, 19-28 (2019).

23. Murray, P. T. et al. Potential use of biomarkers in acute kidney injury: report and summary of recommendations from the 10th Acute Dialysis Quality Initiative consensus conference. Kidney Int. 85, 513-521 (2014).

24. Selby, N. M. et al. An organizational-level program of intervention for AKI: a pragmatic stepped wedge cluster randomized trial. J. Am. Soc. Nephrol. 30, 505-515 (2019).

25. Kellum, J. A. \& Prowle, J. R. Paradigms of acute kidney injury in the intensive care setting. Nat. Rev. Nephrol. 14, 217-230 (2018).

26. Sarma, A., Calfee, C. S. \& Ware, L. B. Biomarkers and precision medicine: state of the art. Crit. Care Clin. 36, 155-165 (2020).

27. Endre, Z. H. et al. Differential diagnosis of AKI in clinical practice by functional and damage biomarkers: workgroup statements from the tenth Acute Dialysis Quality Initiative Consensus Conference. Contrib. Nephrol. 182, 30-44 (2013).

28. McCullough, P. A. et al. Implementation of novel biomarkers in the diagnosis, prognosis, and management of acute kidney injury: executive summary from the tenth consensus conference of the Acute Dialysis Quality Initiative (ADQI). Contrib. Nephrol. 182, 5-12 (2013).

29. Stanski, N. L. et al. PERSEVERE biomarkers predict severe acute kidney injury and renal recovery in pediatric septic shock. Am. J. Respir. Crit. Care Med. 201, 848-855 (2020).

30. Wong, H. R. et al. A Multibiomarker-Based Model for Estimating the Risk of Septic Acute Kidney Injury. Crit. Care Med 43, 1646-1653 (2015).

31. Morrell, E. D., Kellum, J. A., Pastor-Soler, N. M. \& Hallows, K. R. Septic acute kidney injury: molecular mechanisms and the importance of stratification and targeting therapy. Crit. Care 18, 501 (2014).

32. Clark, E. \& Bagshaw, S. M. Long-term risk of sepsis among survivors of acute kidney injury. Crit. Care 18, 103 (2014).

33. Sykes, L., Nipah, R., Kalra, P. \& Green, D. A narrative review of the impact of interventions in acute kidney injury. J. Nephrol. 31, 523-535 (2018).

34. Bagshaw, S. M. Acute kidney injury care bundles. Nephron 131, 247-251 (2015).

35. Hoste, E. A. \& De Corte, W. Implementing the kidney disease: improving global outcomes/acute kidney injury guidelines in ICU patients. Curr. Opin. Crit. Care 19, 544-553 (2013).

36. Koyner, J. L., Zarbock, A., Basu, R. K. \& Ronco, C. The impact of biomarkers of acute kidney injury on individual patient care. Nephrol. Dial. Transplant. 35, 1295-1305 (2020).

37. Talisa, V. B., Yende, S., Seymour, C. W. \& Angus, D. C. Arguing for adaptive clinical trials in sepsis. Front. Immunol. 9, 1502 (2018).

38. Sims, C. R., Nguyen, T. C. \& Mayeux, P. R. Could biomarkers direct therapy for the septic patient? J. Pharm. Exp. Ther. 357, 228-239 (2016).

39. Basu, R. K. et al. Incorporation of biomarkers with the renal angina index for prediction of severe AKI in critically ill children. Clin. J. Am. Soc. Nephrol. 9, 654-662 (2014).

40. Basu, R. K. et al. Identification of candidate serum biomarkers for severe septic shock-associated kidney injury via microarray. Crit. Care 15, R273 (2011).

41. Wong, H. R. et al. Prospective clinical testing and experimental validation of the Pediatric Sepsis Biomarker Risk Model. Sci. Transl. Med. 11, 518 (2019).

42. Shankar-Hari, M. \& Rubenfeld, G. D. Population enrichment for critical care trials: phenotypes and differential outcomes. Curr. Opin. Crit. Care 25, 489-497 (2019). 